\title{
A localized basis that allows fast and accurate second order Møller-Plesset calculations
}

\author{
Joseph E. Subotnik* and Martin Head-Gordon* ${ }^{* \dagger}$
}

October 22, 2004

\begin{abstract}
We present a method for computing a basis of localized orthonormal orbitals (both occupied and virtual), in whose representation the Fock matrix is extremely diagonal-dominant. The existence of these orbitals is shown empirically to be sufficient for achieving highly accurate MP2 energies, calculated according to Kapuy's method. This method (which we abbreviate KMP2), which involves a different partitioning of the $n$-electron Hamiltonian, scales at most quadratically with potential for linearity in the number of electrons. As such, we believe the KMP2 algorithm presented here could be the basis of a viable approach to local correlation calculations.
\end{abstract}

${ }^{*}$ Department of Chemistry, University of California, Berkeley, and Chemical Sciences Division, Lawrence Berkeley National Laboratory, Berkeley, CA, 94720.

†To whom correspondence should be addressed: mhg@cchem.berkley.edu 


\section{Introduction}

Computational chemists have long recognized that in order to efficiently describe electron correlation for large systems, one must employ local occupied and virtual orbitals and exploit the predominantly local nature of electronic excitations. Many past methods have been proposed to do this and much progress has been made in the last two decades towards designing better and better local-correlation algorithms. In this paper, we will focus on local MP2 (second order Møller-Plesset) algorithms.

The predominant school of thought today was pioneered by Pulay and coworkers[1], who suggested focusing only on the "non-negligibly" correlated pairs of occupied orbitals and truncating the virtual space corresponding to each such localized occupied pair. This general strategy of Pulay's was later combined with pseudo-spectral techniques[2, 3] for computational efficiency. Most recently, Schutz, Werner et al have made further improvements to yield linear scaling local algorithms for MP2.[4, 5] The Schutz and Werner algorithms have been very successful at computing LMP2 energies for large systems and the linear-scaling of the current algorithms is quite impressive. The only limitation of these Pulay-inspired approaches is that by correlating only certain chosen domains, one produces non-continuous potential energy surfaces $[6]$. This makes geometric minimization and transition-state determination difficult.

A second school of thought for local correlation limits the allowed substitutions according to atomic criteria (without any cutoffs), and produces an energy which is an entirely differentiable function of the nuclear coordinates. [7, $8,9,10]$ However, thus far, this approach has not scaled better than $N^{3}$, where $N$ is the size of the basis. The primary obstacle for this technique has been handling non-orthogonal, redundant functions, which are necessary to localize all orbitals around atoms.

In considering the achievements and limitations of the local correlation algorithms above, one concludes that in an ideal world, the perfect localcorrelated approach should satisfy four conditions:

1. The computational time needed by the algorithm should scale linearly with system.

2. The algorithm should employ only non-redundant, orthonormal basis functions which have clear quantum mechanical interpretations as oneelectron states and whose use simplifies all future calculations.

3. The local-correlation energy should be a smooth function of the nuclear coordinates. 
4. The expression for the local correlation energy should not contain any unphysical constants with values fixed by empirical parametrization.

Currently, no local-correlation method has been able to satisfy all of the above requirements. However, there is much promise that this will be achieved in the future, whether by a Pulay-like method, an atom-centered method, or Laplace-MP2-like method.[11]

In this paper, we offer our own new local-correlation algorithm which scales at most quadratically and with potential for linear scaling, works only with localized orthonormal orbitals, is a differentiable function of the nuclear coordinates, and whose only drawback is two empirically fixed parametersalbeit these parameters have clear physical meaning.

Our paper revisits the work of Ede Kapuy has experimented over the last two decades with different partitions of the full $n$-electron Hamiltonian in order to introduce locality into many body perturbation theory (MBPT). $[12,13,14,15,16,17,18]$ In particular, Kapuy's approach was to consider the Fock operator in a basis of localized orthonormal occupied and virtual orbitals, and then set the primary Hamiltonian as the diagonal piece of the Fock operator in this basis. Kapuy then treated the off-diagonal elements of the Fock-matrix and the two-electron coulomb term together as the joint perturbation in MBPT.

The advantage of Kapuy's approach is that one may compute energies in a local basis (rather than canonical basis), which allows for better scaling. Furthermore, by his choice of primary Hamiltonian with known eigenvectors, Kapuy could write down the second-order perturbation energy explicitly, without needing to compute any amplitudes recursively (as in the case of normal Pulay-Saebo LMP2[1]).

The disadvantage of Kapuy's method, however, is that the primary Hamiltonian is now somewhat farther from the true Hamiltonian, a defect which slows down and even questions the convergence of a perturbation expansion. After all, the success of canonical MP2 theory is predicated on the premise that the Fock matrix is close to the full $n$-electron Hamiltonian, and that the two-electron Coulumb terms are small in comparison to the Fock matrix. However, for Kapuy's method to work well, we must also suppose that the off-diagonal elements of the Fock matrix in a local basis are small compared to the diagonal elements of the Fock matrix-and this is not generally true. Though we do expect the Fock matrix to be band diagonal in a local representation (because electrons should interact primarily locally), we do not expect the off-diagonal terms to be orders of magnitude smaller than the diagonal elements. Indeed, nearest neighbors don't interact neglibily in 
chemistry! Kapuy himself recognized this limitation of his approach from the very beginning. For good results, Kapuy and those who followed him usually went to third, fourth, or higher order perturbations (see section 3) to reach reasonably converged perturbative energies for chemical species.

However, higher order perturbation theory is expensive. In his seminal paper of 1983, Kapuy recognized that in order for his approach to be widely applicable and useful, "Cheap localization procedures should be developed which can satisfy the double requirement: well localized orbitals with small off-diagonal Fock matrix elements." [12] To our knowledge, this idea has not yet been pursued, and is the basic idea behind this paper.

\section{Balancing Locality With Diagonality of the Fock Matrix}

Our approach towards constructing an orthonormal basis of occupied and virtual orbitals, which are both local and for which the Fock matrix is diagonaldominant, is by construction of a new function designed to intermediate the Boys' function and the sum of the squares of the non-diagonal terms of the Fock matrix. In other words, we construct and minimize the function:

$$
f\left(\eta_{1}, \ldots, \eta_{n}\right)=\sum_{i=1}^{n}<\eta_{i}\left|\left(\hat{\mathbf{r}}-<\eta_{i}|\hat{\mathbf{r}}| \eta_{i}>\right)^{2}\right| \eta_{i}>+\lambda \cdot \sum_{i \neq j=1}^{n}\left|<\eta_{i}\right| \hat{F}\left|\eta_{j}>\right|^{2}
$$

or alternatively, using the invariance of the Trace, we maximize

$$
f\left(\eta_{1}, \ldots, \eta_{n}\right)=\sum_{i=1}^{n}\left|<\eta_{i}\right| \mathbf{r}\left|\eta_{i}>\right|^{2}+\lambda \cdot \sum_{i=1}^{n}\left|<\eta_{i}\right| \hat{F}\left|\eta_{i}>\right|^{2}
$$

In equation (2) above, $\lambda$ is a real parameter designed to weight how much we stress Boys locality (through the first term) and how much we stress the near-diagonality of the Fock Matrix (the second term). $\sqrt{1 / \lambda}$ has the units of force. For $\lambda=0$, we recover the Boys' orbitals; for $\lambda \rightarrow \infty$, we recover the canonical (delocalized) molecular orbitals. Of course, because we intend to localize the occupied and virtual spaces separately, we note that we actually have two parameters to work with: $\lambda_{o}$ and $\lambda_{v}$. In this paper, we have performed numerical calculations for different values of $\lambda_{o}$ and $\lambda_{v}$, and examined the trade-off between locality and diagonal-dominance of the Fock matrix. We find that this trade-off is not severe and that both conditions can be met well enough simultaneously such that MP2 energies can be calculated 
accurately and with a computational time which scales quadratically (or potentially linearly).

Before offering more details, we note at this point that in all calculations below involving the occupied space, we worked only with the valence occupied space. We performed a separate Boys' localization on the core orbitals.

\section{$2.1 \quad$ Occupied Orbitals}

The function described above has been implemented into a development version of the Q-Chem program[19]. In Figure 1a, we plot, as a function of $\lambda_{o}$, both the sum squared of the variances of the corresponding occupied orbitals and the sum squared of the off-diagonal elements of the corresponding Fock matrix (in the occupied-occupied block). The conclusion here is that for moderately sized $\lambda_{o}$, i.e. $\lambda_{o}<50$, the orbitals are not very different from $\lambda_{o}=0$, the Boys orbitals. For that reason, we choose below to focus on $\lambda_{o}=5.0$, giving us just a small correction to the Boys' orbitals. Future work may focus on larger values of $\lambda_{o}$, but we have focused here only on small corrections to the Boys' orbitals. Note that the graph in Figure 1a does not approach the canonical molecular orbitals. In Figure 2, we show three-dimensional pictures of one occupied orbital for $\lambda_{o}=0,10,10^{2}, 10^{3}, 10^{4}, 10^{5}, \infty$. In order to achieve $\max _{i \neq j}\left|F_{i j}\right|<0.01$, one must choose $\lambda_{o}>40,000$.

\section{$2.2 \quad$ Virtual Orbitals}

The situation above with occupied orbitals contrasts strongly with the case of the virtual orbitals. In Figure $1 b$, we plot, as a function of $\lambda_{v}$, both the sum squared of the variances of the corresponding virtual orbitals and the sum squared of the off-diagonal elements of the corresponding Fock matrix (in the virtual-virtual block). We see that for relatively small $\lambda_{v}$, there is a precipitous drop in the sum of the squares of the off-diagonal elements of the Fock matrix. In other words, we can relax the Boys orbitals in such a way that the resulting orbitals, while still local and similar to the Boys orbitals, are much closer to mean-field solutions of the full Hamiltonian. Hence, these relaxed orbitals are expected to be far better suited to perturbation theory (and other local-correlation) calculations. This need for orbital relaxation when doing local MP2 calculations is made clear below. Again, note that the graph in Figure 1b does not approach the canonical molecular orbitals. In Figure 3, we show three-dimensional pictures of one virtual orbital for $\lambda_{v}=0,10,10^{2}, 10^{3}, 10^{4}, 10^{5}, \infty$. In order to achieve $\max _{a \neq b}\left|F_{a b}\right|<0.01$, one must choose $\lambda_{v}>80,000$. 


\section{$3 \quad$ KMP2 Energies of Localized Orbitals}

In canonical MP2 theory, one partititions the true $n$-electron Hamiltonian (H) as

$$
\mathbf{H}=\mathbf{F}+\alpha \cdot(\mathbf{H}-\mathbf{F})
$$

where $\mathbf{F}$ (the Fock operator) is the unperturbed Hamiltonian, $\mathbf{H}-\mathbf{F}$ is the perturbation, and $\alpha=1$. Standard perturbation theory tells us that the second-order perturbative (MP2) energy is:

$$
E_{M P 2}=\sum_{i j a b} \frac{\left|<\chi_{i} \chi_{j}\right|\left|\chi_{a} \chi_{b}>\right|^{2}}{F_{i i}+F_{j j}-F_{a a}-F_{b b}}
$$

where here we sum over the canonical occupied spin-orbitals $\chi_{i}, \chi_{j}$ and the canonical virtual spin-orbitals $\chi_{a}, \chi_{b}$. The canonical spin-orbitals arise here because they are the eigenvectors of the Fock matrix.

Of course, the computational problem with canonical MP2 theory is that it is very slow asymptotically. If $o$ and $v$ are the number of occupied and virtural orbitals respectively, one must compute $o^{2} v^{2}$ integrals before computing the MP2 energy. Construction of these integrals scales as $N^{5}$, where $N$ is the size of the basis. The price is prohibitive for large systems. The inherent difficulty is clearly that the canonical orbitals are delocalized and one must compute all $o^{2} v^{2}$ integrals for accuracy.

According to the Kapuy formalism, one introduces locality into the equations and speeds up an MP2 calculation by choosing a different partioning of the Hamiltonian. One does this by finding a localized set of both occupied and virtual orbitals $\left\{\eta_{i}, \eta_{a}\right\}$, transforming the Fock matrix into this basis, and then choosing the diagonal pieces of the Fock matrix as the unperturbed Hamiltonian. More formally, one writes

$$
\begin{aligned}
\mathbf{F} & =\mathbf{F}^{d}+\mathbf{F}^{n d} \\
\mathbf{F}^{d} & =\sum_{i} F_{i i}\left|\eta_{i}><\eta_{i}\right|+\sum_{a} F_{a a}\left|\eta_{a}><\eta_{a}\right| \\
\mathbf{F}^{n d} & =\sum_{i \neq j} F_{i j}\left|\eta_{i}><\eta_{j}\right|+\sum_{a \neq b} F_{a b}\left|\eta_{a}><\eta_{b}\right| \\
\mathbf{H} & =\mathbf{F}^{d}+\beta \cdot \mathbf{F}^{n d}+\gamma \cdot(\mathbf{H}-\mathbf{F})
\end{aligned}
$$

Now we have two perturbations atop our unperturbed Hamiltonian, and we label these two perturbations by the parameters $\beta$ and $\gamma$. Again, in the end we always take $\beta=\gamma=1$.

The equations for a double pertubation are very simple to compute in principle. One merely expands the wavefunction in a Taylor series of both 
$\beta$ and $\gamma$, and solves term by term equating coefficients. More formally, one defines

$$
\begin{array}{r}
\left|\Psi>=\sum_{m, n=0}^{\infty} \beta^{m} \gamma^{n}\right| \Psi^{(m, n)}> \\
E=\sum_{m, n=0}^{\infty} \beta^{m} \gamma^{n} E^{(m, n)}
\end{array}
$$

and sets $\mathbf{H}|\Psi>=E| \Psi>$. Here $\mid \Psi^{(0,0)}>$ and $E^{(0,0)}$ are the unperturbed eigenvector and eigenvalue of the unperturbed Hamiltonian (i.e. the HartreeFock ground state and Hartree-Fock energy usually).

At high orders (i.e. $n, m \geq 3$ ), these equations become complicated as the $\beta$ and $\gamma$ equations couple. However, the most important correction is the second-order correction in $\gamma$ (i.e. $\mathbf{H}-\mathbf{F}$ ), for which there is a simple explicit expression:

$$
E_{K M P 2}=E^{(0,2)}=\sum_{i j a b} \frac{\left|<\eta_{i} \eta_{j}\right|\left|\eta_{a} \eta_{b}>\right|^{2}}{F_{i i}+F_{j j}-F_{a a}-F_{b b}}
$$

Henceforward, we shall call this energy the KMP2 correction. We reserve the term MP2 for the standard perturbative correction using the canonical MO's as our basis and the full Fock matrix as the primary Hamiltonian (i.e. eqn(4)). Note that when $\eta_{i}, \eta_{a}$ are localized, the KMP2 correction should be computable in at most quadratic, or potentially linear time, for one need only consider orbitals close to each other. This will be discussed further below.

As stated in the introduction, past work on Kapuy's MPBPT theory in local orbitals have always required the addition of third and higher order perturbative corrections in order to account for the off-diagonal elements of the Fock matrix. In other words, Kapuy et al[12, 13, 14, 18] have focused on computing the quantity

$$
e_{L}^{(2)}=E^{(0,2)}+E^{(1,2)}+E^{(2,2)}+\cdots+E^{(L, 2)}
$$

for large values of $L$, and comparing the resulting quantity to the standard MP2 energy correction.

However, in this paper, we focus exclusively on the $E^{(0,2)}$ perturbative correction, given by eq. (7). We have explored whether one can find a localized set of orbitals for which $E^{(0,2)} \approx E_{M P 2}$. Our hypothesis has been that such orbitals do roughly exist, provided one uses a defining function of the form given by eqn (2), which simultaneously localizes orbitals and minimizes their off-diagonal Fock matrix elements. 
We do admit from the outset that our goal is not crystal clear: after all, how large a fraction of the MP2 energy must be recovered for this method to be successful? The answer depends on how accurate an energy is required by the chemist. One notes that canonical MP2 calculations themselves do not yield the most accurate molecular energies when compared to more complete correlation treatments, and one should not look to either canonical MP2 or Kapuy-MP2 for extremely accurate correlation energies. After all, the entire validity of canonical MP2 is predicated on the assumption that the Fock matrix is a good approximation to the true $n$-electron Hamiltonian, an assumption which can be valid only to a certain degree. This point is discussed in more detail in section 3.4. With this reservation, we present below $E_{K M P 2}$ and $E_{M P 2}$ energies from many different localized orbitals, and the reader may determine for himself whether this method recovers enough of the MP2 energy for his own satisfaction. At the very least, the reader will observe from our data that one can find localized orbitals which are much better energetically than standard Boys or Pipek-Mezey orbitals. These orbitals may well find use as starting guesses in other local-correlation techniques. At best, the reader may be content with this method as a good and cheap substitute for local MP2 calculations.

One word should be said at this point about the types of orbitals analyzed below. Clearly, there are infinitely many sets of localized orbitals, and one could in principle generate useful orbitals from balancing Fock diagonaldominance with any of the Edmiston-Ruedenberg or Pipek-Mezey or Boys criteria. Future work may well find an advantage to using other types of localization for KMP2 corrections, but of the three listed above, we have chosen the Boys orbitals because they have a more sparse set of exchange integrals than Pipek-Mezey, and they are computationally cheaper than EdmistonRuedenberg orbitals and just as sparse[20]. (Here we focus on sparsity of the exchange matrix because that is the ultimate criteria for speeding up KMP2 calculations.)

\subsection{KMP2 energy as a function of $\lambda$}

In section 2, we showed that one could reduce the off-diagonal elements of the Fock matrix (in a localized basis) moderately by allowing a nonzero value of $\lambda_{o}$ and drastically by allowing a nonzero value of $\lambda_{v}$. We now show that this reduction in the size of the off-diagonality of the Fock matrix translates into a significant improvement in the KMP2 correlation energy. In Tables $1 a$ and $1 b$, we list KMP2 energies as a function of $\lambda_{o}$ and $\lambda_{v}$.

Tables $1 a$ and $1 b$ show that relaxation of the Boys orbitals is crucial towards attaining accurate KMP2 energies. Interestingly, in the case of pen- 
tane, relaxing the occupied orbitals is not as crucially important as relaxing the localized virtual orbitals. On the one hand, using the canonical virtual orbitals, one gains only $2.5 \%$ of the MP2 energy by relaxing the Boys occupied orbitals with increasing values of $\lambda_{o}$. On the other hand, using the canonical occupied orbitals, one gains only $25 \%$ of the MP2 energy by relaxing the Boys virtual orbitals with increasing values of $\lambda_{v}$. In this paper we have focused on $\lambda_{v}=40$, as we sought the smallest correction to the Boys virtual orbitals at which relaxation was significant. Again, future work may focus on larger values of $\lambda_{v}$.

\subsection{Dependence on Basis Set}

Regarding the dependence of energies on basis set, Pipek and Bogar showed[18] that the Boys orbitals give a decent KMP2 correlation energy in a STO-3G basis ( $97.7 \%$ of the canonical MP2 energy)-however, this energy becomes worse as the basis set grows to $6-31 G^{*}(75 \%)$. This result is plausible because, in a very small basis set, there are very few virtual orbitals, and these orbitals are forced to be far from each other and not very interacting. Hence, the Fock matrix does not have big off-diagonals and no relaxation of localization is necessary. However, in a bigger basis set, many virutal orbitals are located close to each other in space, interacting strongly, and the Fock matrix in the Boys orbital representation is not very diagonal-dominant. In Table 2, we show the amount of MP2 energy recovered by our local method for increasing basis set size. We conclude that, though the Boys orbitals give worse and worse KMP2 energies as the basis set grows, the $(5,40)$ basis does not do much worse. In other words, the parameter $\lambda_{v}=40$ may be applicable even beyond the $6-31 \mathrm{G}^{*}$ basis. See the discussion below.

\subsection{Scaling With Alkanes of Increasing Size}

The most important requirement that we require of our basis of localized

orbitals and KMP2 calculations are that they scale correctly with increazing size. After all, the strength of working in a local basis should be that one can work with ever bigger systems. In this paper, for concreteness, we worked with alkanes of increasing length. Our essential requirements were:

1. As the length of the alkane chain grew, our method should capture a constant percentage of the MP2 energy.

2. Because the KMP2 calculations are sped up by the sparsity of the matrix elements $(i a \mid i a)$, where $i$ is an occupied orbital and $a$ is a virtual orbital, we required that the number of significant $i a$ pairs, i.e. those 
pairs with $(i a \mid i a)>1.2 \times 10^{-7}$, should be proportional to the size of the system. Without locality, i.e. for canonical orbitals, the number of significant ( $i a \mid i a)$ pairs scale as the square of the size of the system.

In Tables $3 a$ and $3 b$, we show that our KMP2 method does indeed capture a constant percentage of MP2 correlation energy for increasing system sizes; furthermore, the number of significant $i$ a pairs grows linearly, as we wanted. For comparison, we also show in Table $3 b$ that the sparsity of the $(i a \mid i a)$ matrix for the Boys orbitals is not much more than the sparsity for the $(5,40)$ orbitals. Thus, for the large systems, we should be able to calculate accurate MP2 energies quickly with orbitals almost as localized as the Boys orbitals.

\subsection{Atomization Energies for G2 data}

As the benchmark for measuring the accuracy of our method, we calculated the atomization energies per bond for the 148 molecules in the neutral G2-1 data set[21]. All calculations were done using a 6-31G* basis set and an MP2 perturbative correction on top of an unrestricted HF solution. We always set $\lambda_{v, \uparrow}=\lambda_{v, \downarrow}$ and $\lambda_{o, \uparrow}=\lambda_{o, \downarrow}$. The results are in Table (4). Empirically, we find that for larger atoms (e.g. $\mathrm{Cl}, \mathrm{Si}$ ), higher values of $\lambda_{o}$ are necessary to correctly match MP2 energy calculations. This result can be rationalized by observing that the lone pairs on bigger atoms are more diffuse, and their centroids can be separated more than those lone pairs on a smaller atom. Hence, the Boys function value is bigger, and we need to provide a larger $\lambda_{o}$ value. For this reason, we list energies for $\lambda_{o}=0,5,40$ and we divide the G2 data set into molecules with large and small atoms (Table $4 a$ and Table $4 b$ ).

From the data in the third column of Table 4, we conclude again that relaxation of the Boys orbitals is crucial towards using local orbitals efficiently in an MP2 setting. Furthermore, the atomization energies per bond recorded here show the strength and potential of the KMP2 approach. For the molecules in the G2 set, KMP2 usually captures $90-100 \%$ of the MP2 atomization energy. Although the atomization energies of this set of molecules can be computed at best to within an accuracy of 1.25-3.00 kcal/mol using our choice of finite parameters, we should recall that canonical MP2 itself cannot compute truly accurate atomization energies to within $3 \mathrm{kcal} / \mathrm{mol}$ either. Jung et al[22] have recently computed $E_{M P 2}$ and $E_{Q C I S D(T)}$ for 77 molecules drawn mostly from the G2 data set. From this data set, one com-

putes $\frac{\sqrt{\left(E_{M P 2}-E_{Q C I S D T)^{2}}\right.}}{\text { Number of Bonds }}=5.1 \mathrm{kcal} / \mathrm{mol}$, implying that our $\left(E_{K M P 2}-E_{M P 2}\right)$ error is smaller than the error inherent in MP2. Hence, while our KMP2 approach does suffer clear systematic error relative to MP2, this error is small 
enough that KMP2 may find direct use in future calculations. Or at the very least, as we have stressed above, the KMP2 orbitals will also likely be used as initial guesses for more accurate iterative correlation energies.

\section{The Hylleraas Function and Higher-Order Corrections}

One may improve upon the $E_{K M P 2}$ energy by invoking the Hyllerass function[23], which is a variational function for the MP2 energy defined in terms of scattering amplitudes $t_{i j a b}$. The Hylleraas function has the form:

$$
L(\mathbf{t})=\frac{1}{4}(\mathbf{t} \cdot \mathbf{A} \cdot \mathbf{t}+2 \mathbf{t} \cdot \mathbf{I})
$$

where

$$
\begin{aligned}
A_{i j a b, k l c d} & =\left(F_{a c} \delta_{b d}+F_{b d} \delta_{a c}\right) \delta_{i k} \delta_{j l}-\left(F_{i k} \delta_{j l}+F_{j l} \delta_{i k}\right) \delta_{a c} \delta_{b d} \\
I_{i j a b} & =<\eta_{i} \eta_{j} \| \eta_{a} \eta_{b}>
\end{aligned}
$$

Clearly, then, we must solve the equation

$$
\mathbf{A} \cdot \mathbf{t}=-\mathbf{I}
$$

The strength of the Hylleraas approach is that, when the guess amplitudes are of order $\epsilon$ from the correct MP2 amplitudes, the guess energy is of order $\epsilon^{2}$ from the correct MP2 energy. Now because $A$ is diagonal dominant in the $(5,40)$ basis, we take for our guess amplitudes

$$
t_{i j a b}^{(0)}=\frac{<\eta_{i} \eta_{j} \| \eta_{a} \eta_{b}>}{F_{i i}+F_{j j}-F_{a a}-F_{b b}}
$$

using the Kapuy orbitals defined here for various values of $\lambda_{o}, \lambda_{v}$. In other words, we write $A=A_{\text {diag }}+A_{\text {nondiag }}$ and we guess $\mathbf{t}^{(0)}=\mathbf{A}_{\text {diag }}^{-1} \cdot \mathbf{I}$. We then immediately plug these amplitudes into the Hylleraas function $L(\mathbf{t})$. Given the sparsity of the $t_{i j a b}^{(0)}$ amplitudes, this computation should still scale with order $N^{2}$ or even $N$. The only disadvantage of computing the Hylleraas energy is that one must make and store all of the $\left\langle\eta_{i} \eta_{j}\right| \eta_{a} \eta_{b}>$ integrals (which is not necessary to compute $E_{K M P 2}$.

In Table 4 below, in the fourth column, we present the results of Hylleraas calculations. As we expected, the error in the atomization energies is much smaller for the Hylleraas function than for $E_{K M P 2}$. Thus, the Hylleraas 
energy is certainly a viable alternative and improvement to $E_{K M P 2}$. We plan to investigate the computational scaling in more detail in the future.

Besides the Hylleraas function, one can calculate the third order $E^{(1,2)}$ correction in order to more accurately compute the correlation energy. This piece was often measured in the past by Kapuy and coworkers both as a correction and as a means to assess convergence of the pertubative expansion. The $E^{(1,2)}$ correction can written in many different forms, including:

$E^{(1,2)}=\sum_{i j a b} \sum_{k l c d} \frac{<\Psi_{H F}|\mathbf{H}-\mathbf{F}| \Psi_{i j}^{a b}><\Psi_{i j}^{a b}\left|\mathbf{F}^{n d}\right| \Psi_{k l}^{c d}><\Psi_{k l}^{c d}|\mathbf{H}-\mathbf{F}| \Psi_{H F}>}{\left(E_{H F}-E_{i j a b}\right)\left(E_{H F}-E_{k l c d}\right)}$

This correction can also be written down as the natural result after one round of Pulay-Saebo amplitude iteration, as follows:

1. Set $\mathbf{t}^{(0)}$ according to equation 8 .

2. Construct $\mathbf{t}^{(1)}=\mathbf{A}_{\text {diag }}^{(-1)}\left(-\mathbf{I}-\mathbf{A}_{\text {nondiag }} \mathbf{t}^{(0)}\right)$.

3. $E^{(1,2)}=\left(\mathbf{t}^{(1)}-\mathbf{t}^{(0)}\right) \cdot \mathbf{I}$. We may define $e_{1}^{(2)}=E^{(0,2)}+E^{(1,2)}=\mathbf{t}^{(1)} \cdot \mathbf{I}$.

This method presumably has a higher cost than evaluating the Hylleraas energy for it requires an iteration that scales formally as $N^{5}$ before sparsity. The computational cost of this iteration will surely be reduced given the sparsity of the $t_{i j a b}$ amplitudes. However, it appears more difficult to make this algorithm quadratic or linear scaling than it is for the Hylleraas function; in particular, this algorithm should be more sensitive to the decay of the Fock matrix with distance. Regarding energy accuracy, we expected both the Hylleraas energy and the $e_{1}^{(2)}$ energy to perform well given our optimized orbitals. On the one hand, the $e_{1}^{(2)}$ energy is correct to third order and thus should be more accurate than the Hylleraas energy; on the other hand, of the two methods discussed here, only the Hylleraas energy is variational and this also has advantages. For a good comparison of these methods, we show in Table 4 the size of the $E^{(0,2)}$ correction and the accuracy of $e_{1}^{(2)}$ energy. One notes that the $E^{(1,2)}$ correction is in general small in a good basis. We conclude that the KMP2 energy is generally not far from the MP2 energy, that one may improve significantly upon the $E_{K M P 2}$ energy by calculating $E^{(1,2)}$, but that the Hylleraas function is probably the fastest and easiest method for calculating a cheap and more accurate perturbative correlation energy. 


\section{Discussion}

The results in this paper are the natural continuation of the proposal made by Kapuy in 1983. The orbitals constructed here seemingly satisfy locality and Fock diagonal-dominance, the double criteria called for by Kapuy more than twenty years ago. Furthermore, the results of this paper suggest that an attractive trade-off between accuracy and speed may exist within the KMP2 framework. However, we caution the reader that more numerical testing is needed to confirm the wide applicability of this method. Furthermore, we certainly do not claim here that $\left(\lambda_{o}=5, \lambda_{v}=40\right)$ is the optimal choice of parametrization. We have demonstrated this choice is satisfactory in a 6-31G* basis in certain cases, e.g. alkane chains, but future work may well decide on a different optimal set of parameters. Likely, this choice will depend on the atoms involved. As stated previously, larger atoms seem to require larger values of $\lambda_{o}$. Certainly, in the future, more work is needed to determine more rigorously how $\lambda_{v}$ should change with basis size.

Regarding the optimal defining function for the Kapuy orbitals, we repeat that we have not made an exhaustive study of all the different localization criteria. In fact, it is plausible that future work may even decide not to use the Boys' criteria at all, instead using the Pipek-Mezey or Edmiston-Ruedenberg or other functional forms. On a philosophical level, there is the deep question of how can we characterize all of the different sets of localized orbitals, such that we can search through them, looking for those with smallest off-diagonal Fock elements? On a practical level, if we believe that our final function will be the sum of a localizing term and a Fock-diagonalizing term, as is eqn (2), then our goal should be to find the localization criteria which gives the orbitals (occupied and virtual) with the most sparse exchange integrals, for this is the critera used to enhance the scaling of most algorithms.

At this point, we should mention that one problem with the current defining function for the optimized Kapuy orbitals in this paper is that, as $\lambda$ grows very large, the defining function takes on multiple maxima. In other words, the contours of the defining function are rugged for $0 \ll \lambda \ll \infty$. Of course, the uniqueness of the Kapuy orbitals as the unique stationary point of a defining function is not crucial at all for our purposes. Our only concern is that we different researchers should be able to reliably and consistenly determine the same Kapuy orbitals for different calculations. To ensure that is the case, in this paper, we have always chosen the global maxima of the defining function, but we expect that a better and more stable algorithm to be found in the future for quickly and precisely determining either the local or global maximum of our defining function (and hence the optimized Kapuy orbitals). 
The main result of this paper, namely that one can relax the Boys orbitals to minimize the off-diagonal Fock elements while maintaining locality, should have implications for other local correlation methods. After all, the $(5,40)$ orbitals represent local one-electron states which are "not far" from canonical solutions. Might these orbitals find use as good initial guesses for other local correlation techniques, including local coupled-cluster approaches? We are currently investigating this point.

In summary, much work still remains both to find the optimum orbitals for this method and to resolve all of the implications of a $\lambda \neq 0$ relaxation. Moreover, in the future, a low-scaling version of this algorithm needs to be implemented for others to use. Notwithstanding these limitations, the Kapuy-MP2 method should scale quadratically or even linearly, uses only orthonormal orbitals, smoothly varies with nuclear coordinates, and has exactly two or four paramters. These attributes make it an attractive starting point for future local correlation work.

\section{Conclusion}

In this paper, we have presented an elementary approach towards localizing molecular orbitals while keeping the Fock-matrix close to diagonal. We have further demonstrated the utility of these orbitals in calculating near accurate and fast KMP2 energies. Furthermore, our algorithm is a smooth function of nuclear coordinates, and hence can be used in geometric optimizations. We expect that, in the future, either this procedure should find direct use in quantum mechanical calculations of large molecules or that our simple method should be a benchmark algorithm which other, more sophisticated algorithms need to out-perform.

\section{Acknowledgments}

JES was supported by the Fannie and John Hertz Foundation. This work was supported by a grant from the Computational Nanosciences Program of the Department of Energy. MHG is a part owner of Q-Chem.

\section{References}

[1] Saebo, S. and Pulay, P. Annu. Rev. Phys. Chem. 44, 213 (1993). 
[2] Murphy, R.B., Beachy, M.D., Friesner, R.A. and Ringnalda, M.N. J. Chem. Phys. 103, 1481 (1995).

[3] Reynolds,G., Martinez,T. and Carter,E. J. Chem. Phys. 105, 6455 (1996).

[4] Schutz,M., Hetzer, G. and Werner, H.J. J. Chem. Phys. 111, 5691 (1999).

[5] Werner, H.J., Manby, F.R., and Knowles, P.J. J. Chem. Phys. 118, 8149 (2003).

[6] Russ,N. and Crawford, T.D. J. Chem. Phys. 121, 691 (2004).

[7] Head-Gordon, M., Maslen, P.E. and White, C.A. J. Chem. Phys. 108, 616 (1998).

[8] Maslen, P.E. and Head-Gordon, M. J. Chem. Phys. 109, 7093 (1998).

[9] Lee, M.S., Maslen, P.E. and Head-Gordon, M. J. Chem. Phys 112, 3592 (2000).

[10] Maslen, P.E., Dutoi, A.D., Lee, M.S., Shao, Y.H. and Head-Gordon, M. Mol. Phys. (submitted)

[11] Ayala, P.Y. and Scuseria, G.E. J. Chem. Phys. 110, 3660 (1999).

[12] Kapuy, E., Csepes, Z. and Kozmuta, C. Int. J. Quant. Chem. 23, 981 (1983).

[13] Kapuy, E., Bartha, F., Bogar, F., Csepes, Z., and Kozmutza, C. Int. J. Quant. Chem. 38, 139 (1990).

[14] Kapuy, E., Bogar, F., and Kozmutza, C. J. Mol Struc. 297, 365 (1993).

[15] Kapuy, E., Bogar, F., and Tfirst, E. Int. J. Quant. Chem. 52, 127 (1994).

[16] Kozmutza, C., Kapuy, E., Evleth, E., and Kassab, E. J. Mol. Struc 332, 141 (1995).

[17] Kozmutza, C., Kapuy, E., Evleth, E., Pipek, J., and Trezl, L. Int. J. Quant. Chem. 57, 775 (1996).

[18] Pipek, J. and Bogar, F. Topics Curr. Chem. 203, 43 (1999).

[19] J. Kong et al. J. Comp. Chem, 21, 1532 (2000). 
[20] Joe Subotnik, unpublished data.

[21] Curtiss, L., Raghavachari, K., Trucks, G. and Pople, J. J. Chem. Phys. 94, 7221 (1991).

[22] Jung, Y.,Lochan, R., Dutoi, A. and Head-Gordon, M. Accepted for publication in J. Chem. Phys..

[23] Kutzelnigg, W. Theor. Chim. Acta. 68, 445 (1985). 


\section{Figures}

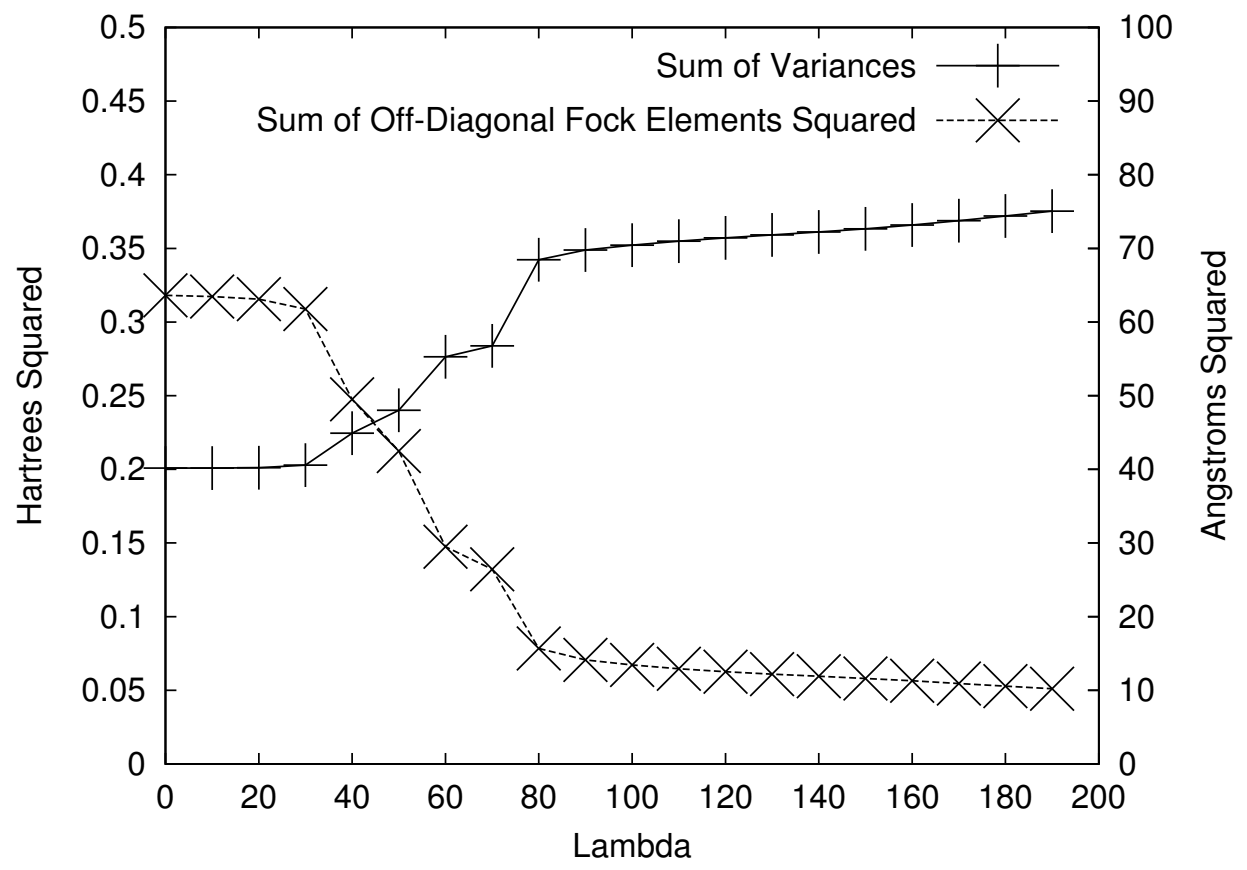

Figure 1a: The sum of the variances squared $\left(\AA^{2}\right)$ and the sum of the offdiagonal Fock matrix elements squared (Hartrees ${ }^{2}$ ) versus $\lambda_{o}$ for the occupied space of $\mathrm{C}_{5} \mathrm{H}_{12}$. For the canonical molecular orbitals, these numbers are $\sum_{i} v_{i}^{2}=302 \AA^{2}$ and $\sum_{i \neq j} F_{i j}^{2}<1.0 \times 10^{-14}$ Hartrees $^{2}$ respectively. The basis is 6-31G*. 


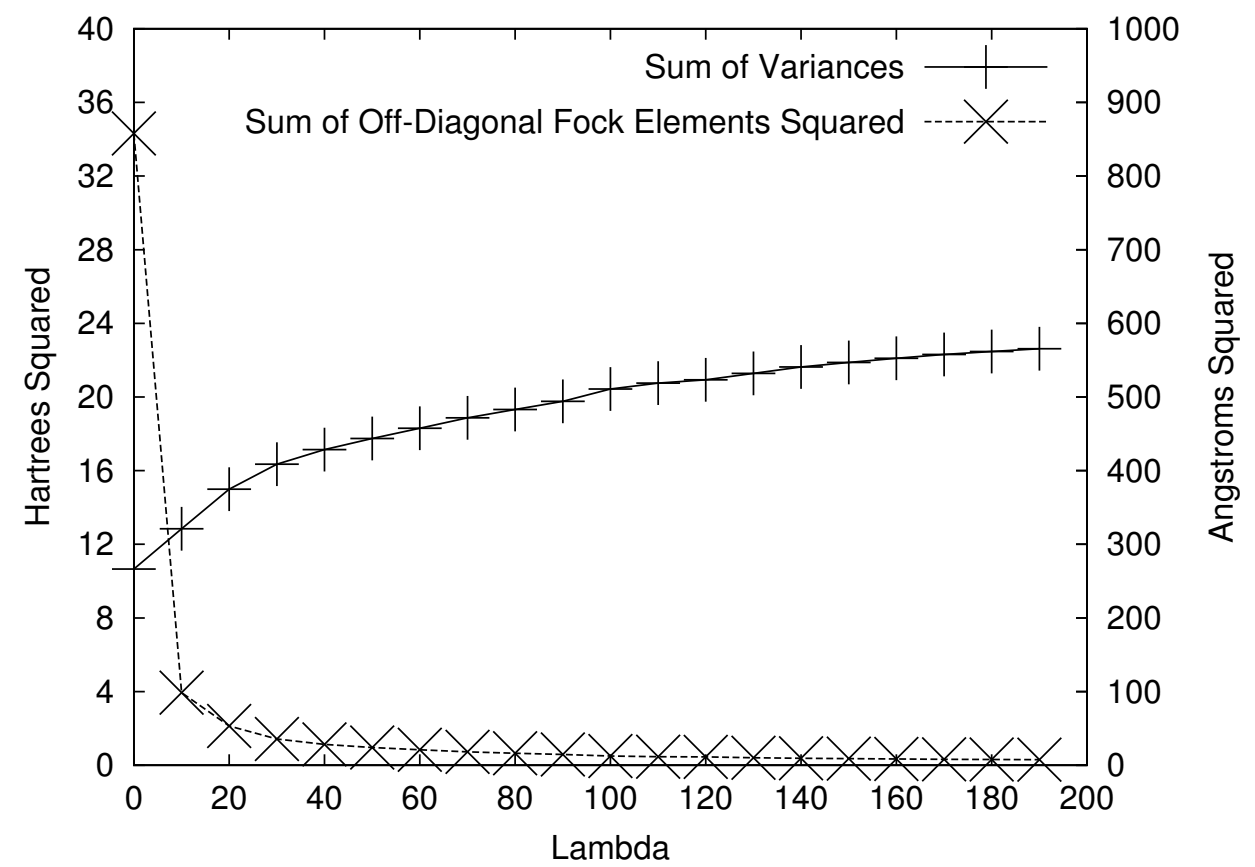

Figure 1b: The sum of the variances squared $\left(\AA^{2}\right)$ and the sum of the offdiagonal Fock matrix elements squared (Hartrees ${ }^{2}$ ) versus $\lambda_{v}$ for the virtual space of $C_{5} H_{12}$. For the canonical molecular orbitals, these numbers are $\sum_{i} v_{i}^{2}=1585 \AA^{2}$ and $\sum_{i \neq j} F_{i j}^{2}<1.0 \times 10^{-14}$ Hartrees $^{2}$ respectively. Note the precipitous drop in the Fock off-diagonal term for $\lambda_{v}>0$. The basis is 6-31G*. 


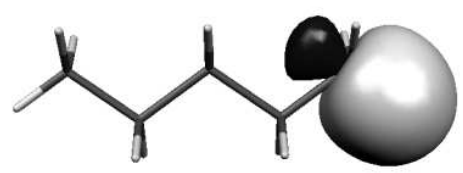

Figure 2a:

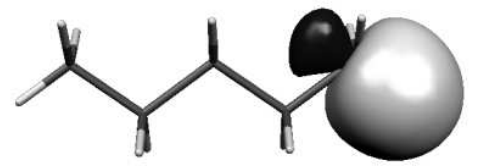

Figure 2b:

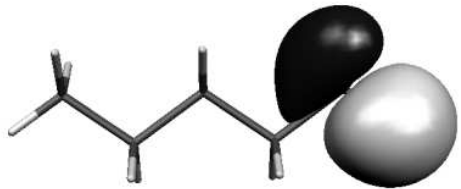

Figure 2c:

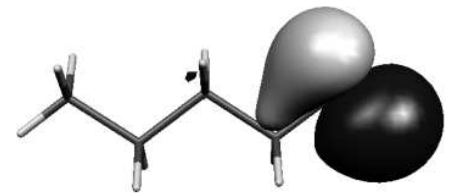

Figure 2d:

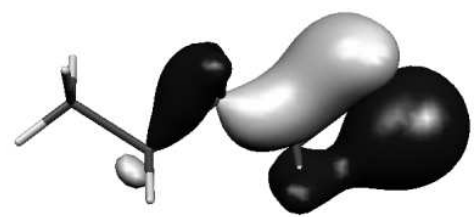

Figure 2e:

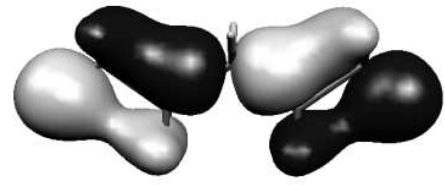

Figure 2f:

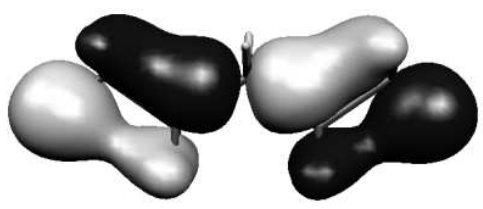

Figure 2g:

Figure 2: One occupied orbital of $C_{5} H_{12}$ in a $6-31 \mathrm{G}^{*}$ basis. $\lambda_{o}=(a) 0,(b) 10,(c) 10^{2},(d) 10^{3},(e) 10^{4},(f) 10^{5},(g) \infty$. 


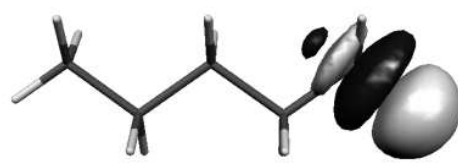

Figure 3a:

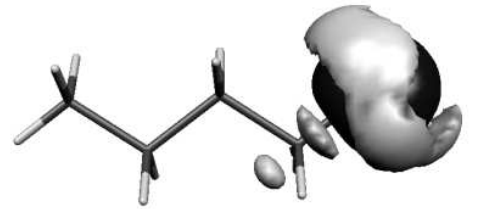

Figure 3b:

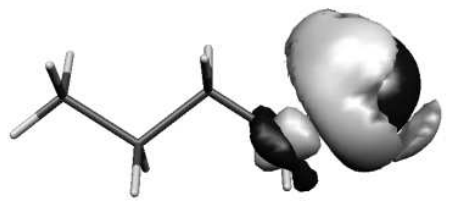

Figure 3c:

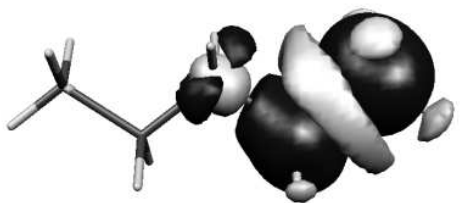

Figure 3d:

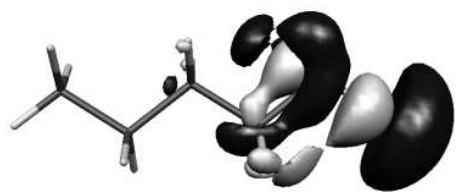

Figure 3e:

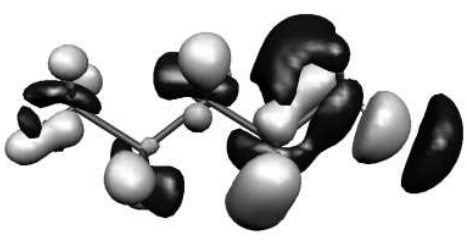

Figure 3f:

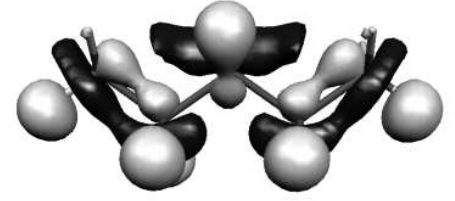

Figure 3g:

Figure 3: One virtual orbital of $C_{5} H_{12}$ in a $6-31 \mathrm{G}^{*}$ basis.

$$
\lambda_{v}=(a) 0,(b) 10,(c) 10^{2},(d) 10^{3},(e) 10^{4},(f) 10^{5},(g) \infty .
$$




\section{Tables}

\begin{tabular}{|c|c|}
\hline$\lambda_{o}$ & KMP2/MP2 \\
\hline 0 & $97.5 \%$ \\
\hline 10 & $97.5 \%$ \\
\hline 20 & $97.5 \%$ \\
\hline 30 & $97.4 \%$ \\
\hline 40 & $97.7 \%$ \\
\hline 50 & $98.0 \%$ \\
\hline 60 & $98.7 \%$ \\
\hline 70 & $98.7 \%$ \\
\hline 80 & $99.8 \%$ \\
\hline 90 & $99.8 \%$ \\
\hline
\end{tabular}

Table 1a: The percentage of the MP2 correlation energy for $C_{5} H_{12}$ captured by our KMP2 method versus the size of $\lambda_{o}$. Here, $\lambda_{v}=\infty$. The relaxation is noticeable here, but not drastic as in Table $1 \mathrm{~b}$. The basis is 6-31G*.

\begin{tabular}{|c|c|}
\hline$\lambda_{v}$ & KMP2/MP2 \\
\hline 0 & $75.1 \%$ \\
\hline 10 & $93.9 \%$ \\
\hline 20 & $97.2 \%$ \\
\hline 30 & $98.6 \%$ \\
\hline 40 & $99.5 \%$ \\
\hline 50 & $99.8 \%$ \\
\hline 60 & $100 \%$ \\
\hline 70 & $100 \%$ \\
\hline 80 & $100 \%$ \\
\hline 90 & $100 \%$ \\
\hline
\end{tabular}

Table 1b: The percentage of the MP2 correlation energy for $C_{5} H_{12}$ captured by our KMP2 method versus the size of $\lambda_{v}$. Here, $\lambda_{0}=\infty$. Note the importance of relaxation here. Furthermore, note that we are close to the infinite limit for $\lambda_{v}=40$. The basis is $6-31 \mathrm{G}^{*}$. 


\begin{tabular}{|c|c|c|c|}
\hline $\begin{array}{c}\text { Basis } \\
\text { Set }\end{array}$ & Dimension & $\begin{array}{c}\lambda_{o}=0 \\
\lambda_{v}=0 \\
\text { KMP2/MP2 }\end{array}$ & $\begin{array}{c}\lambda_{o}=5 \\
\lambda_{v}=40 \\
\text { KMP2/MP2 }\end{array}$ \\
\hline sto-3g & 37 & $97.7 \%$ & $97.7 \%$ \\
\hline $6-31 \mathrm{G}^{*}$ & 99 & $74.0 \%$ & $97.0 \%$ \\
\hline 6-31G** & 135 & $66.3 \%$ & $96.9 \%$ \\
\hline $6-311 \mathrm{G}^{* *}$ & 162 & $63.5 \%$ & $94.1 \%$ \\
\hline 6-311+G** & 182 & $63.6 \%$ & $94.3 \%$ \\
\hline $6-311++\mathrm{G}^{* *}$ & 194 & $64.2 \%$ & $94.5 \%$ \\
\hline cc-pVTZ & 318 & $53.8 \%$ & $94.4 \%$ \\
\hline
\end{tabular}

Table 2: The percentage of the MP2 correlation energy for $C_{5} H_{12}$ captured by our KMP2 method versus basis set size. Here, we provide data for the Boys orbitals $\lambda_{v}=\lambda_{o}=0$ and the $(5,40)$ orbitals. Note that the Boys orbitals get worse and worse as the basis set increases, while the $(5,40)$ set has a near constant effectiveness. 


\begin{tabular}{|c|c|c|c|}
\hline $\mathrm{n}$ & $\begin{array}{c}\text { True MP2 Correlation } \\
\text { energy (Hartrees) }\end{array}$ & $\begin{array}{c}(5,40) \text { KMP2 } \\
\text { energy (Hartrees) }\end{array}$ & $\begin{array}{c}\text { Percent of MP2 } \\
\text { energy captured }\end{array}$ \\
\hline 10 & -1.31755 & -1.27377 & $96.7 \%$ \\
\hline 20 & -2.63159 & -2.53890 & $96.5 \%$ \\
\hline 30 & -3.94563 & -3.80133 & $96.3 \%$ \\
\hline 40 & -5.25967 & -5.07004 & $96.3 \%$ \\
\hline
\end{tabular}

Table 3a: The percentage of the MP2 correlation energy captured by our KMP2 method versus alkane size. Here, $n$ stands for $C_{n} H_{2 n+2}$. The basis is 6-31G*.

\begin{tabular}{|c|c|c|c|c|c|}
\hline $\mathrm{n}$ & $\begin{array}{c}\text { Total number } \\
\text { of } i a \text { pairs }\end{array}$ & $\begin{array}{c}\text { Number of } \\
\text { significant } \\
\text { ia pairs } \\
\text { Boys Orbs. }\end{array}$ & $\begin{array}{c}\text { Percent } \\
\text { significant } \\
\text { ia pairs } \\
\text { Boys Orbs. }\end{array}$ & $\begin{array}{c}\text { Number of } \\
\text { significant } \\
\text { ia pairs } \\
(5,40) \text { Orbs. }\end{array}$ & $\begin{array}{c}\text { Percent } \\
\text { significant } \\
\text { ia pairs } \\
(5,40) \text { Orbs }\end{array}$ \\
\hline 10 & 4743 & 3581 & $75.5 \%$ & 3711 & $78.2 \%$ \\
\hline 20 & 18483 & 8362 & $45.2 \%$ & 8662 & $46.9 \%$ \\
\hline 30 & 41223 & 13101 & $31.8 \%$ & 13623 & $33.1 \%$ \\
\hline 40 & 72963 & 17907 & $24.5 \%$ & 18435 & $25.3 \%$ \\
\hline
\end{tabular}

Table 3b: The number of significant $i a$ pairs, where here $i$ is an occupied orbital and $a$ is a virtual orbitals. We define $i a$ to be significant if $(i a \mid i a)>1.2 \times 10^{-7}$. Here, $n$ stands for $C_{n} H_{2 n+2}$. The basis is $6-31 \mathrm{G}^{*}$. 


\begin{tabular}{|c|c|c|c|c|c|}
\hline$\lambda_{o}$ & $\lambda_{v}$ & $\begin{array}{c}\text { RMSD } \\
\left(E_{K M P 2}^{a t}-E_{M P 2}^{a t}\right)\end{array}$ & $\begin{array}{c}\text { RMSD } \\
\left(E_{H y l}^{a t}-E_{M P 2}^{a t}\right)\end{array}$ & $\begin{array}{c}\text { RMSD } \\
E_{m o l}^{(1,2)}-E_{\text {atoms }}^{(1,2)}\end{array}$ & $\begin{array}{c}\text { RMSD } \\
e_{1}^{(2), a t}-e_{1}^{(2), a t}\end{array}$ \\
\hline 0 & 0 & 17.08 & 9.84 & 10.17 & 7.08 \\
\hline 5 & 40 & 2.43 & 0.64 & 2.11 & 0.35 \\
\hline 40 & 40 & 1.26 & 0.47 & 1.03 & 0.26 \\
\hline 0 & $\infty$ & 4.38 & 1.54 & 3.55 & 0.85 \\
\hline 5 & $\infty$ & 2.10 & 0.53 & 1.84 & 0.28 \\
\hline 40 & $\infty$ & 0.87 & 0.29 & 0.72 & 0.14 \\
\hline
\end{tabular}

Table 4a: The errors in atomization energy per bond of the neutral G2 data set calculated in a 6-31G* basis for different values of $\lambda_{o}, \lambda_{v}$. Here we considered only those chemical species of the G2 data set without any atoms in the third row of the Periodic Table. This includes 98 molecules of the 148 total. All atom-atom interactions are treated as one bond each. For instance, $\mathrm{CH}_{4}$ has four bonds in these calculations, and $\mathrm{CO}_{2}$ has two bonds. Here $E^{a t}$ stands for atomization energy. We report the RMS difference in atomization energy per bond for the KMP2 expression (column 3), for the Hylleraas energy (column 4), and for the third order once-iterated $e_{1}^{(2)}$ expression (column 6). Column 5 contains the RMSD for the third order correction $E^{(1,2)}$ calculated per bond. See text for more details. Energies are given in $\mathrm{kcal} / \mathrm{mol}$.

\begin{tabular}{|c|c|c|c|c|c|}
\hline$\lambda_{o}$ & $\lambda_{v}$ & $\begin{array}{c}\text { RMSD } \\
\left(E_{K M P 2}^{a t}-E_{M P 2}^{a t}\right)\end{array}$ & $\begin{array}{c}\text { RMSD } \\
\left(E_{H y l}^{a t}-E_{M P 2}^{a t}\right)\end{array}$ & $\begin{array}{c}\text { RMSD } \\
E_{m o l}^{(1,2)}-E_{\text {atoms }}^{(1,2)}\end{array}$ & $\begin{array}{c}\text { RMSD } \\
e_{1}^{(2), a t}-e_{1}^{(2), a t}\end{array}$ \\
\hline 0 & 0 & 17.73 & 10.14 & 10.56 & 7.32 \\
\hline 5 & 40 & 4.97 & 1.65 & 4.14 & 0.89 \\
\hline 40 & 40 & 3.00 & 1.23 & 2.43 & 0.65 \\
\hline 0 & $\infty$ & 4.97 & 1.65 & 4.18 & 0.81 \\
\hline 5 & $\infty$ & 3.53 & 1.05 & 3.09 & 0.49 \\
\hline 40 & $\infty$ & 1.44 & 0.54 & 1.26 & 0.21 \\
\hline
\end{tabular}

Table 4b: The error in atomization energy per bond of the neutral G2 data set calculated in a $6-31 \mathrm{G}^{*}$ basis for different values of $\lambda_{o}, \lambda_{v}$. Here we considered only those chemical species of the G2 data set with at least one atom in the third row of the Periodic Table. This includes 50 molecules of the 148 total. All atom-atom interactions are treated as one bond each. For instance, $C_{C l}$ has four bonds in these calculations, and $O C S$ has two bonds. Here $E^{a t}$ stands for atomization energy. We report the RMS difference in atomization energy per bond for the KMP2 expression (column 3), for the Hylleraas energy (column 4), and for the third order once-iterated $e_{1}^{(2)}$ expression (column 6). Column 5 contains the RMSD for the third order correction 
$E^{(1,2)}$ calculated per bond. See text for more details. Energies are given in $\mathrm{kcal} / \mathrm{mol}$. 